\title{
MATHEMATICAL MODELING
} МАТЕМАТИЧЕСКОЕ МОДЕЛИРОВАНИЕ

УДК 519.226

\author{
В.Р. Крашенинников, Ю.Е. Кувайскова, О.Е. Маленова, А.Ю. Субботин \\ ПСЕВДОГРАДИЕНТНЫЙ АЛГОРИТМ ИДЕНТИФИКАЦИИ ДВАЖДЫ
СТОХАСТИЧЕСКОГО ЦИЛИНДРИЧЕСКОГО ИЗОБРАЖЕНИЯ
}

Крашенинников Виктор Ростиславович, доктор технических наук, профессор, окончил Казанский государственный университет, заведующий кафедрой «Прикладная математика и информатика» Ульяновского государственного технического университета. Имеет работы по статистическим методам обработки сигналов и изображений. [e-mail: kvrulstu@тail.ru].

Кувайскова Юлия Евгеньевна, кандидат технических наук, доцент, окончила УлГТУ, доцент кафедры «Прикладная математика и информатика» УлГТУ. Имеет работы по статистическим методам обработки систем временных рядов. [e-mail: u.kuvaiskova@mail.ru].

Маленова Ольга Евгеньевна, окончила УлГТУ, аспирант кафедры «Прикладная математика и информатика» УлГТУ. Имеет работы по статистическим методам обработки сигналов и изображений. [e-mail: nika-lilu@yandex.ru].

Субботин Алексей Юрьевич, окончил УлГТУ, аспирант кафедры «Прикладная математика и информатика» УлГТУ. Ведущий инженер ФНПЦ АО «НПО «Марс». Имеет работы по статистическим методам обработки сигналов и изображений. [e-mail: ashkael@mail.ru].

Аннотация

В настоящее время проблемы обработки изображений становятся все более важными из-за развития систем аэрокосмического мониторинга Земли, радио- и гидролокационных систем, медицинских устройств для ранней диагностики и т. д. Но основная часть работ по их обработке связана с изображениями, определенными на прямоугольных двухмерных сетках или сетках более высокой размерности. В некоторых практических ситуациях изображения определены на цилиндре. Например, изображения участка трубопроводов, кровеносных сосудов, деталей вращения и т. д. Особенность области задания таких изображений требует своего учета в их моделях и алгоритмах обработки. В данной статье рассматриваются авторегрессионные модели цилиндрических изображений, приводятся выражения корреляционной функции в зависимости от параметров авторегрессии. Для представления неоднородных изображений со случайными неоднородностями используются «дважды стохастические» модели, в которых одно или несколько изображений управляют параметрами результирующего изображения. Спиральное сканирование цилиндрического изображения можно рассматривать как квазипериодический процесс из-за корреляции строк изображения. Предложены псевдоградиентные алгоритмы идентификации модели, то есть оценивания параметров скрытых управляющих изображений по заданному результирующему изображению. Статистическое моделирование показало, что эти алгоритмы имеют достаточно высокую точность.

Ключевые слова: цилиндрическое изображение, дважды стохастическая модель, псевдоградиентный алгоритм, квазипериодический процесс, идентификация.

doi: 10.35752/1991-2927-2021-2-64-56-65

1 Исследование выполнено при финансовой поддержке РФФИ в рамках научного проекта № 20-01-00613. 


\section{PSEUDOGRADIENT ALGORITHM FOR IDENTIFICATION OF DOUBLY STOCHASTIC CYLINDRICAL IMAGE}

Viktor Rostislavovich Krasheninnikov, Doctor of Sciences in Engineering, Professor; graduated from Kazan State University; Head of the Department of Applied Mathematics and Informatics of Ulyanovsk State Technical University; an author of articles in the field of statistical methods of signal and image processing. e-mail: kvrulstu@mail.ru.

Iuliia Evgenevna Kuvaiskova, Candidate of Sciences in Engineering, Associate Professor; graduated from Ulyanovsk State Technical University; Associate Professor of the Department of Applied Mathematics and Informatics of UISTU; an author of articles in the field of statistical methods for processing time series systems. e-mail: u.kuvaiskova@mail.ru.

Olga Evgenevna Malenova, Postgraduate Student of the Department of Applied Mathematics and Informatics of Ulyanovsk State Technical University; graduated from UISTU; an author of articles in the field of statistical methods of signal and image processing.e-mail: nika-lilu@yandex.ru.

Aleksei lurevich Subbotin, Postgraduate Student of the Department of Applied Mathematics and Informatics of Ulyanovsk State Technical University; graduated from UISTU; Leading Engineer at FRPC JSC 'RPA 'Mars'; an author of articles in the field of statistical methods of signal and image processing. e-mail: ashkael@mail.ru.

\section{Abstract}

Nowadays image processing problems are becoming increasingly important due to development of the aerospace Earth monitoring systems, radio and sonar systems, medical devices for early diagnosis, etc. However, the most of the image processing works deals with images defined on rectangular two-dimensional grids or grids of higher dimension. In some practical situations images are set on a cylinder, for example images of pipeline sections, blood vessels, rotary parts, etc. The peculiarity of the domain for specifying such images requires its consideration in their models and processing algorithms. The article deals with autoregressive models of cylindrical images and gives some expressions of the correlation function depending on the autoregression parameters are given. To represent heterogeneous images with random heterogeneities, 'doubly stochastic' models are used in which one or more images control the parameters of resulted image. The spiral scan of a cylindrical image can be considered as a quasiperiodic process due to the correlation of image rows. The article proposes the pseudogradient algorithms for the modal identification. The statistical modeling proves these algorithms give good model identification.

Keywords: cylindrical image, doubly stochastic model, pseudogradient algorithm, quasiperiodic process, identification.

\section{ВведенИе}

В настоящее время методы анализа изображений применяются в аэрокосмических системах мониторинга Земли, в системах навигации, радио- и гидролокации, в системах медицинской диагностики и компьютерного зрения, например, [1-4]. Подавляющее большинство работ по обработке изображений имеет дело с изображениями, заданными на прямоугольных двумерных сетках или сетках более высокой размерности [5-9]. Для описания таких изображений используются различные модели, например авторегрессионные, полиномиальные, гиббсовые, канонические разложения и так далее. Работ с изображениями, заданными на сфере и других криволинейных поверхностях, намного меньше, например, [10-13]. Иногда изображения определены на цилиндре, например, изображения трубопровода, кровеносного сосуда, змеи, круглого здания и т. д. Некоторые из цилиндрических изображений показаны на рисунке 1.
Традиционные модели прямоугольных изображений могут использоваться для моделирования только небольших участков таких изображений, а при описании всего изображения модель пришлось бы сильно усложнить, разбивая цилиндр на множество участков.

В разделе 2 данной статьи показано, что хорошо известная модель плоского изображения Хабиби [5] не подходит для описания целого цилиндрического изображения. Его пришлось бы разбивать на множество участков, на каждом из которых можно было бы задать свою модель Хабиби. Такое представление очень неудобно, особенно при дальнейшем синтезе алгоритмов обработки. Поэтому мы предлагаем модель, основанную на одном уравнении авторегрессии для представления всего однородного цилиндрического изображения с использованием цилиндрической спиралевидной сетки. Эту модель можно представить в эквивалентной форме как процесс авторегрессии на спиралевидной 

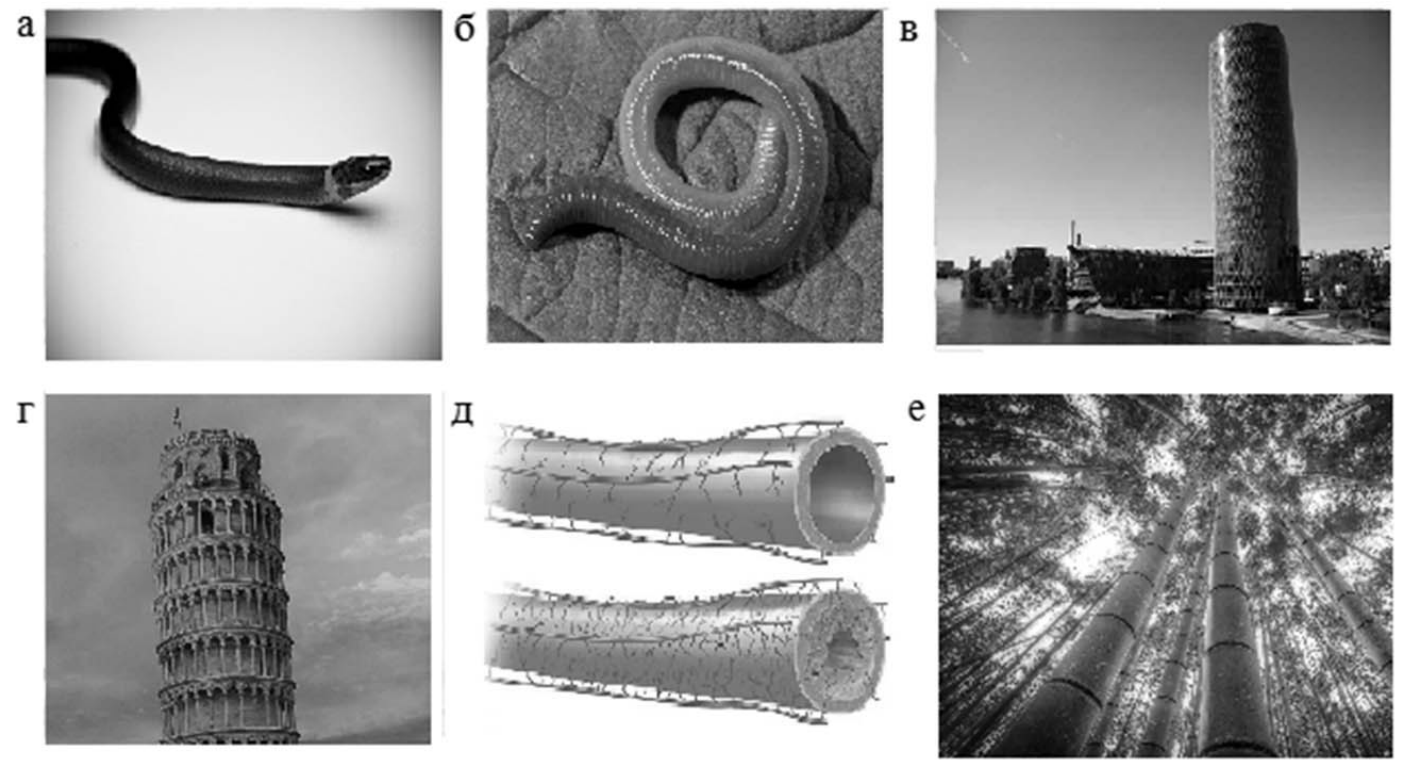

Рис. 1. Примеры цилиндрических изображений: а) змея; б) червь; в) здание; г) Пизанская башня; д) кровеносный сосуд; е) ствол дерева

сетке на том же цилиндре. Это дает возможность представлять квазипериодические случайные процессы. Выражения дисперсии и ковариационной функции даны относительно параметров модели. В разделе 3 «дважды стохастические» модели используются для представления неоднородных изображений со случайными неоднородностями. В этих моделях одно или несколько изображений (управляющих) задают параметры результирующего изображения [13, 14]. Приведены примеры моделирования таких изображений с различными типами управляющих изображений. Управляющие изображения влияют на свойства наблюдаемого результирующего изображения, но сами не наблюдаемы. Таким образом, возникает задача идентификации (оценки) скрытых управляющих изображений. Эта задача решается в разделе 4 с помощью псевдоградиентных адаптивных алгоритмов. Приведены результаты статистических экспериментов с этими алгоритмами, показавшие их достаточно высокую точность.

\section{1 МОДЕЛИ ОДНОРОДНЫХ ИЗОБРАЖЕНИЙ НА ЦИЛИНДРЕ}

Сначала рассмотрим известную авторегрессионную модель

$$
x_{k, l}=a x_{k, l-1}+b x_{k-1, l}-a b x_{k-1, l-1}+\beta \xi_{k, l}
$$

плоского изображения [5], где $k$ - номер строки; $l$ - номер столбца, $\xi_{k, l}$ - независимые стандартные случайные величины.

На рисунке 2а показан пример изображения, смоделированного с помощью этой модели. На рисунке 2в показан график из нескольких строк такого изображения, разделенных вертикальными линиями.

Параметры $a$ и $b$ влияют на горизонтальную и вертикальную корреляции изображения, соответственно, $\beta$ влияет на дисперсию изображения. Сгенерированное
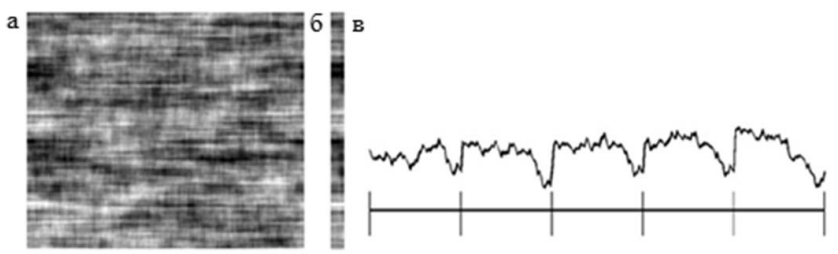

Рис. 2. Изображение, смоделированное (1): а) сгенерированное изображение; б) первые столбцы; в) график смежных строк изображения

изображение имеет нулевое среднее значение и ковариационную функцию (КФ)

$V(m, n)=M\left[x_{k, l} x_{k+m, l+n}\right]=\frac{\beta^{2} a^{|m|} b^{|n|}}{\left(1-a^{2}\right)\left(1-b^{2}\right)}$,

график которой показан на рисунке 3.

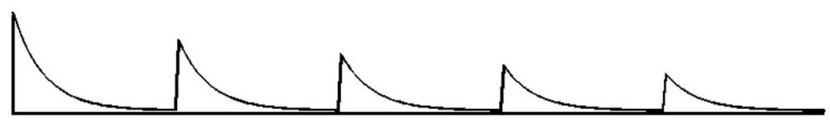

Рис. 3. График КФ модели (1)

Корреляция элементов изображения уменьшается вдоль строк и столбцов. Поэтому элементы этого изображения, расположенные в начале и конце строки, слабо зависимы. При соединении изображения в цилиндр будет большой скачок яркости на стыке (рис. 2a и 26), что не характерно для изображения на цилиндре. Соседние строки прямоугольного изображения с $b \approx 1$ имеют высокую корреляцию. Следовательно, объединяя строки в последовательность, мы можем получить модель квазипериодического процесса. Однако начало и конец каждой строки, находясь на значительном 
расстоянии друг от друга, практически не зависят друг от друга, поэтому на стыке квазипериодов процесса будут резкие скачки (рис. 2в), которые необычны для относительно непрерывных процессов. Таким образом, прямоугольные изображения не дают приемлемого представления цилиндрических изображений и квазипериодических процессов. В данной статье для этой цели используются изображения, заданные на цилиндре, значения которого по спирали не имеют нежелательных резких скачков.
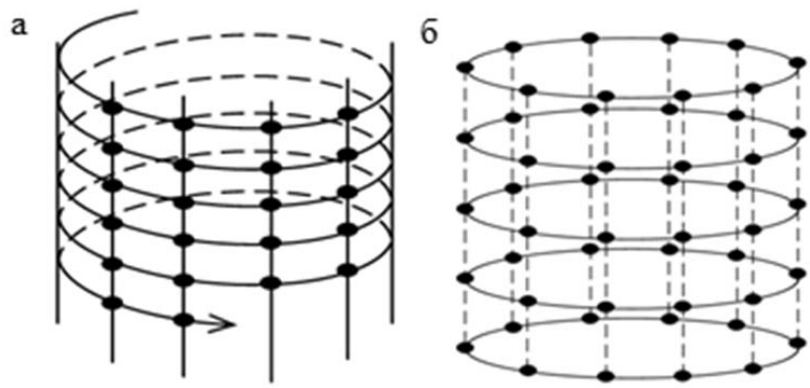

Рис. 4. Представление сетки на цилиндре: а) цилиндрическая сетка; б) круговая сетка

Рассмотрим спиральную сетку на цилиндре (рис. 4а). Строки этой сетки представляют собой витки цилиндрической спирали. Витки этого изображения также можно рассматривать как замкнутые круги на цилиндре с такой же нумерацией (рис. 4б).

Чтобы описать изображение, заданное на цилиндрической сетке, мы используем аналог [11] модели авторегрессии в (1):

$$
x_{k, l}=a x_{k, l-1}+b x_{k-1, l}-a b x_{k-1, l-1}+\beta \xi_{k, l},
$$

где $k$ - номер витка спирали и $l$ - номер узла $(l=0, \ldots, T-1)$ в витке, $T$ - период, то есть количество точек в одном витке.

Эту модель можно представить в виде развертки изображения по спирали:

$$
x_{n}=a x_{n-1}+b x_{n-T}-a b x_{n-T-1}+\beta \xi_{n},
$$

где $n=k T+l$ - номер точки изображения при сквозной нумерации. Можно показать, что КФ этой модели имеет вид:

$$
\begin{array}{r}
V(n)=\beta^{2}\left(\frac{1}{\left(1-b^{2}\right) T} \sum_{k=0}^{T-1} \frac{z_{k}}{\left(1-a z_{k}\right)\left(z_{k}-a\right)} z_{k}^{n}+\right. \\
\left.+\frac{s}{\left(1-a^{2}\right)(1-b s)(s-b)} \rho^{n}\right),
\end{array}
$$

где $z_{k}=\sqrt[T]{b} \exp (i 2 \pi k / T)$ и $s=a^{T}$. Вид графика такой КФ показан на рисунке 5 . Поперечное сечение изображения, полученного с помощью этой модели, показано на рисунке 6. Отметим, что строки на всех следующих рисунках этой статьи представляют соответствующие витки изображения на цилиндре.

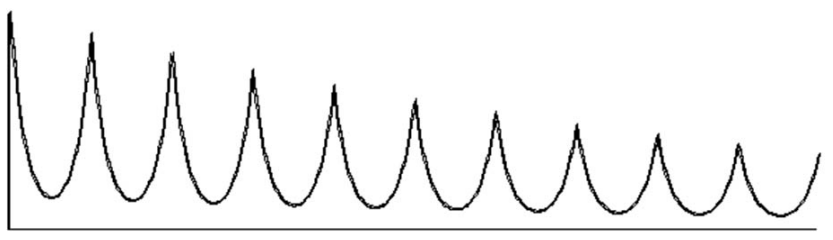

Рис. 5. График КФ процесса, заданный моделью (3)

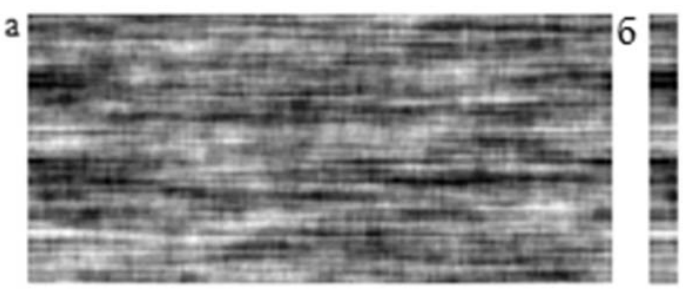

Рис. 6. Цилиндрическое изображение: а) поперечный разрез б) первые столбцы

Характерной чертой КФ (5) является непрерывность на стыке периодов, в отличие от рисунка 3. Изображение на рисунке 6 также непрерывно вдоль линии разреза, что заметно по нескольким первым столбцам (рис. 6б), прикреплённым к этому изображению. В результате процесс, описываемый моделью (4), т. е. развертка цилиндрического изображения по спирали, не имеет резких скачков на стыке периодов (рис. 7). Примеры моделирования цилиндрических изображений при различных значениях параметров этой модели показаны на рисунке 8.

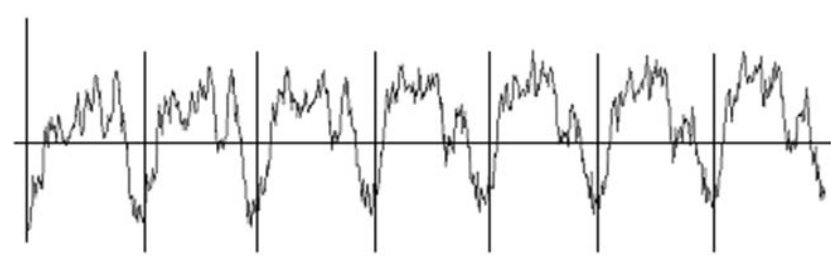

Рис. 7. График моделирования процесса с помощью модели (4)
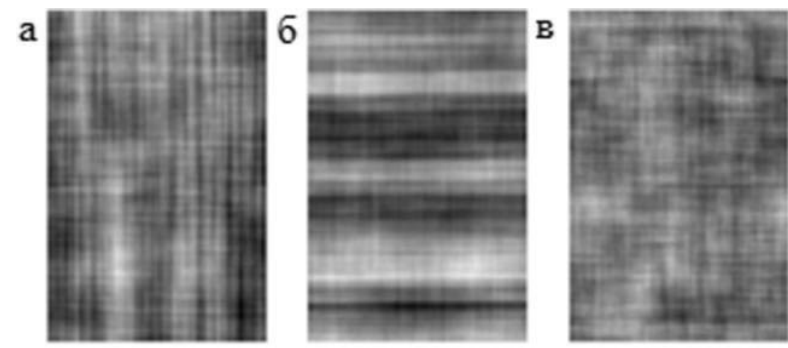

Рис. 8. Смоделированное изображение на цилиндре с параметрами: а) $a=0,95, b=0,99$; б) $a=0,99, b=0,95$; в) $a=b=0,95$

\section{2 МОДЕЛИ СЛУЧАЙНЫХ НЕОДНОРОДНЫХ ИЗОБРАЖЕНИЙ} НА ЦИЛИНДРЕ

Изображения, созданные моделями (3) и (4), являются однородными, что ограничивает область их при- 
менения, поскольку многие изображения и сигналы имеют значительную и случайную неоднородность. В $[13,14]$ предложено представлять неоднородность изображений в виде "дважды стохастической» модели. В этом представлении несколько общих «управляющих» изображений устанавливают случайные параметры для «управляемого» результирующего изображения. Неоднородность получаемого изображения определяется локальными особенностями контрольных изображений. В [14] управляющими и управляемыми изображениями были авторегрессионные изображения, заданные на прямоугольных сетках.

Для представления неоднородных цилиндрических изображений применяется дважды стохастическая модель, аналогичная рассмотренной в [14]. Для этого возьмем два изображения $A=\left\{a_{n}\right\}$ и $B=\left\{b_{n}\right\}$, определим (4) с некоторыми значениями параметров в качестве контрольных изображений. Управляемое изображение $X=\left\{x_{n}\right\}$ также устанавливается (4) с переменными параметрами $a=a_{n} b=b_{n}$.

На рисунке 9 показан пример применения этой модели для имитации изображения на цилиндре. Есть только одно управляющее изображение $(A=B)$, кото-
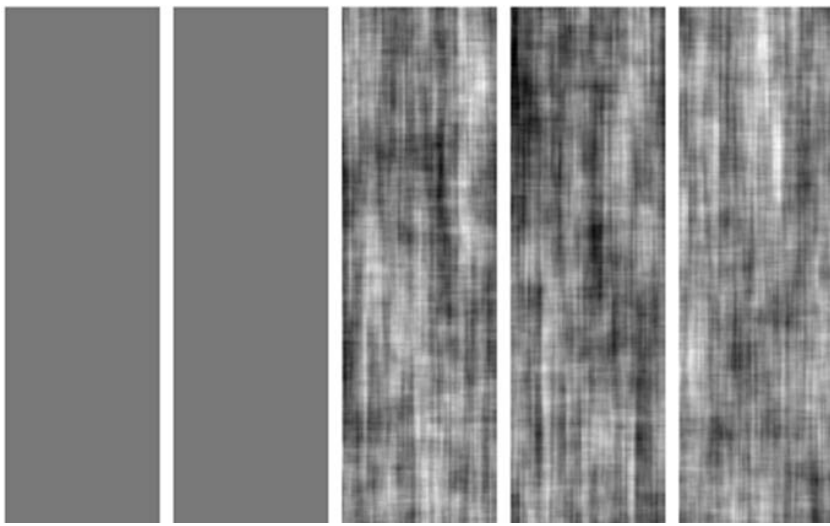

Рис. 10. Моделирование с использованием управляющих изображений Типа 1 с параметрами $a=0,9$, $b=0,98$
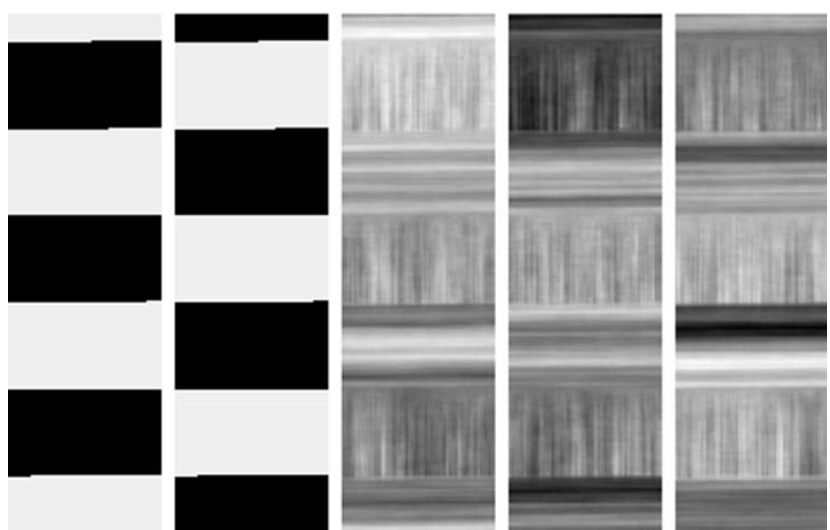

Рис. 11. Моделирование с использованием управляющих изображений Типа 2 с параметрами $a=0,9$, $b=0,8$
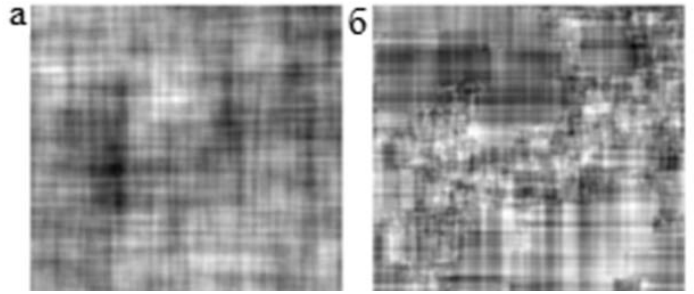

Рис. 9. Пример применения модели (4) для имитации изображения на цилиндре:

а) управляющее изображение; б) управляемое (итоговое) изображение

рое показано на рисунке 9а. Окончательное изображение на рисунке $9 б$ имеет ярко выраженную неоднородность, вызванную как раз изменчивостью параметров модели (4). Темные области на рисунке 9а соответствуют малым значениям $a_{n}$ и $b_{n}$, поэтому в этих местах на рисунке 96 изображение менее коррелировано.

На рисунках 10-13 показаны примеры изображений, сгенерированных с помощью двух управляющих изображений разных типов. Их значения показаны яркостями. Во всех изображениях $T=100$, по 200 строк в каждом. На этих рисунках первые два изображения - это
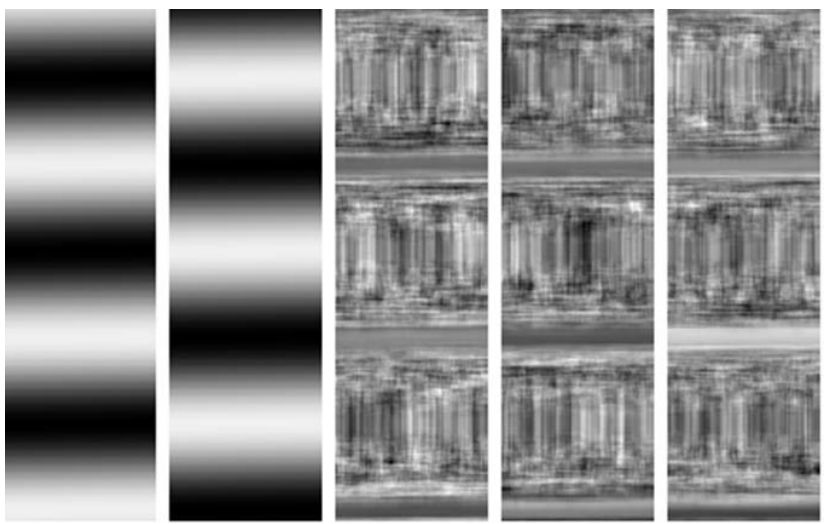

Рис. 12. Моделирование с использованием управляющих изображений Типа 3 с параметрами $a=0,9$, $b=0,8$
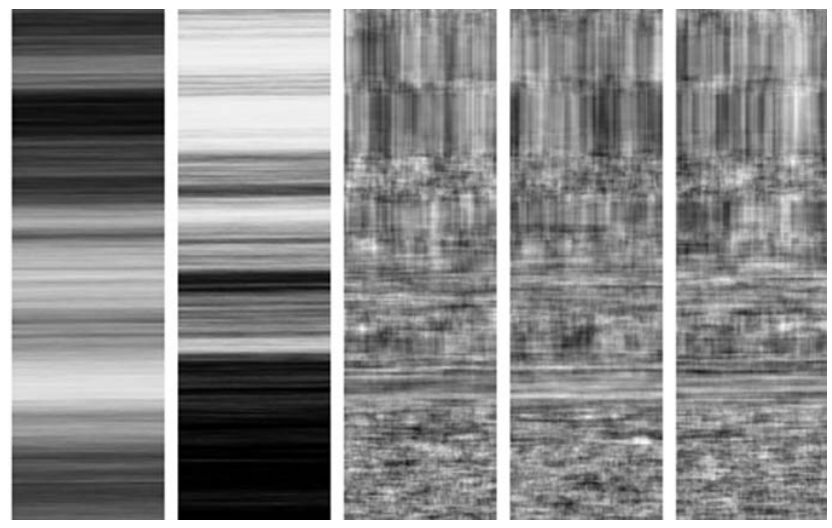

Рис. 13. Моделирование с использованием управляющих изображений Типа 4 с параметрами $a=0,9$, $b=0,8$ 
$A=\left\{a_{n}\right\}$ и $B=\left\{b_{n}\right\}$, последние три изображения это варианты управляемого изображения. Эти три изображения разные из-за различных случайных значений $\xi_{n}$ в (4), но имеют общую структуру из-за одинаковых управляющих изображений.

Рассматриваются конкретные типы управляющих изображений:

Тип 1. Управляющие изображения постоянны: $A=\{a\}$ и $B=\{b\}$.

Тип 2. Управляющие изображения резко меняют значения от $a$ к $b$ и наоборот.

Тип 3. Управляющие изображения гармонически изменяют значения со средними значениями $a$ и $b$.

Тип 4. Управляющие изображения - авторегрессионные со средними значениями $a$ и $b$.

\section{3 ИДЕНТИФИКАЦИЯ УПРАВЛЯЮЩИХ ИЗОБРАЖЕНИЙ}

Скрытые управляющие изображения влияют на структуру результирующего изображения, что необходимо учитывать в алгоритмах его обработки. Кроме того, тип и параметры скрытых изображений могут использоваться для классификации имеющихся реальных изображений. Таким образом, возникает задача идентификации (оценки) скрытых изображений.

Пусть наблюдаемое управляемое изображение $X=\left\{x_{n}\right\}$ задается моделью (4). Требуется оценить скрытые управляющие изображения $A=\left\{a_{n}\right\}$ и $B=\left\{b_{n}\right\}$. Для решения этой задачи мы применим псевдоградиентный $[10,15]$ адаптивный алгоритм

$$
\begin{aligned}
& \hat{a}_{n+1}=\hat{a}_{n}+h \operatorname{sgn}\left[\Delta_{n}\left(x_{n-1}-\hat{b}_{n} x_{n-T-1}\right)\right], \\
& \hat{b}_{n+1}=\hat{b}_{n}+h \operatorname{sgn}\left[\Delta_{n}\left(x_{n-T}-\hat{a}_{n} x_{n-T-1}\right)\right]
\end{aligned}
$$

минимизации

квадратов

ошибок $\Delta_{n}^{2}=\left[x_{n}-\left(\hat{a}_{n} x_{n-1}+\hat{b}_{n} x_{n-T}-\hat{a}_{n} \hat{b}_{n} x_{n-T-1}\right)\right]^{2}$, где $\left(\hat{a}_{n+1}, \hat{b}_{n+1}\right)$ - оценка параметров $(a, b)$, следующая после оценки $\left(\hat{a}_{n}, \hat{b}_{n}\right) ; h$ - значение шага алгоритма. Таким образом, компоненты $\left(\hat{a}_{n+1}, \hat{b}_{n+1}\right)$ отличаются от $\left(\hat{a}_{n}, \hat{b}_{n}\right)$ на $\pm h$.

На рисунке 14 показан пример оценки скрытых изображений типа 3 с параметрами $a=0,6 ; b=0,7 ; T=100$. На рисунках 14а и 14в показаны скрытые управляющие изображения $A$ и $B$, на рисунке 14д показано управляемое изображение $X$ с использованием модели (4). На рисунках 146 и 14г показаны полученные оценки $\hat{A}$ и $\hat{B}$ изображений $A$ и $B$ с использованием (6) с шагом $h=0,001$. Визуально видна хорошая оценка. На рисунках 14е-14к показаны изображения, смоделированные, используя (2) с $\hat{A}$ и $\hat{B}$ в качестве управляющих изображений. Следует отметить, что они схожи с $X$ (рис. 14д). Это означает хорошую оценку скрытых управляющих изображений.

Исследование точности оценок, полученных с помощью алгоритма в (6), проведено статистическим моделированием. Для каждого типа и набора его параметров моделировались скрытые контрольные изображения. На их основе моделировались полученные изображения, к которым применялась процедура оценки параметров контрольных изображений. Было получено более 100000 оценок значений параметров $a$ и $b$ с соответствующим значением $h$, и были рассчитаны среднеквадратичные отклонения (СКО) ошибок по выборке. Результаты представлены на рисунках 15-20 в виде графиков.
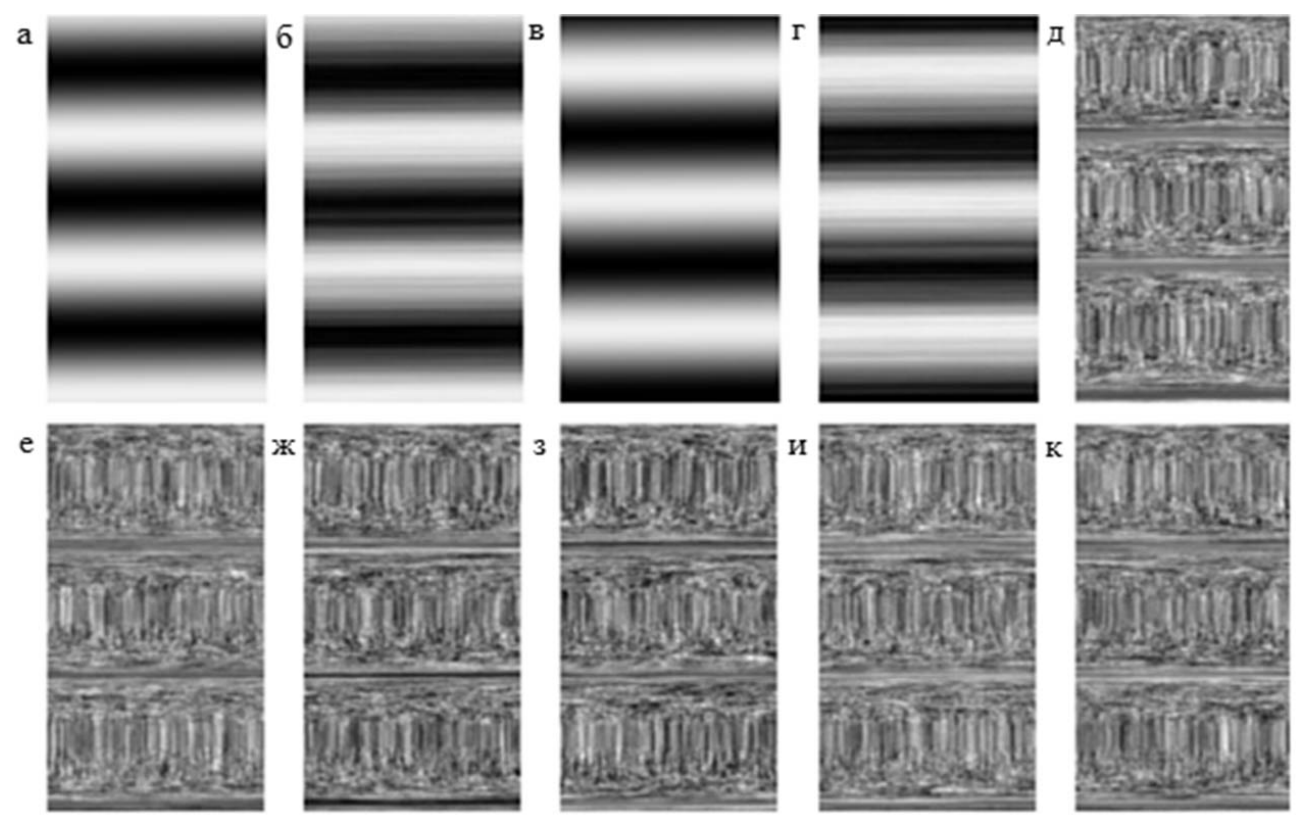

Рис. 14. Пример оценки скрытых изображений Типа 3: а) и в) - это $A$ и $B$; б) и г) - это $\hat{A}$ и $\hat{B}$; д) - изображение, управляемое $A$ и $B$; е)-к) - это изображения, управляемые $\hat{A}$ и $\hat{B}$ 

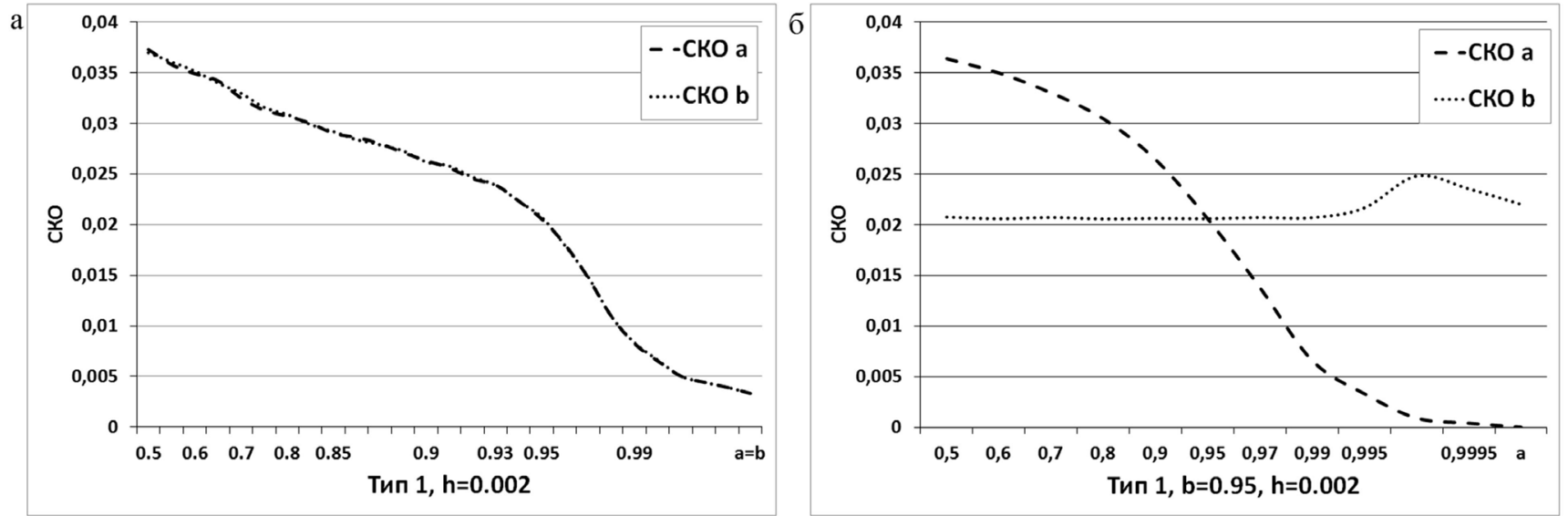

Рис. 15. Тип 1. а) зависимость СКО ошибок от параметров $a=b$; б) зависимость СКО ошибок от параметра $a$
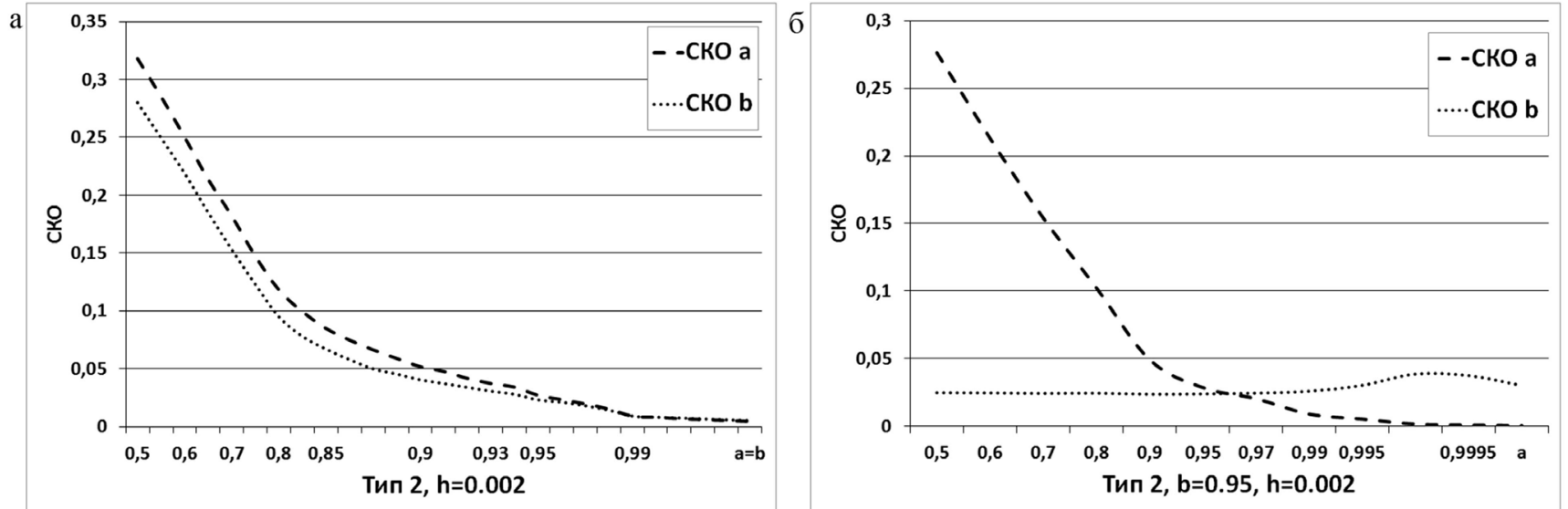

Рис. 16. Тип 2. а) зависимость СКО ошибок от параметров $a=b$; б) зависимость СКО ошибок от параметра $a$
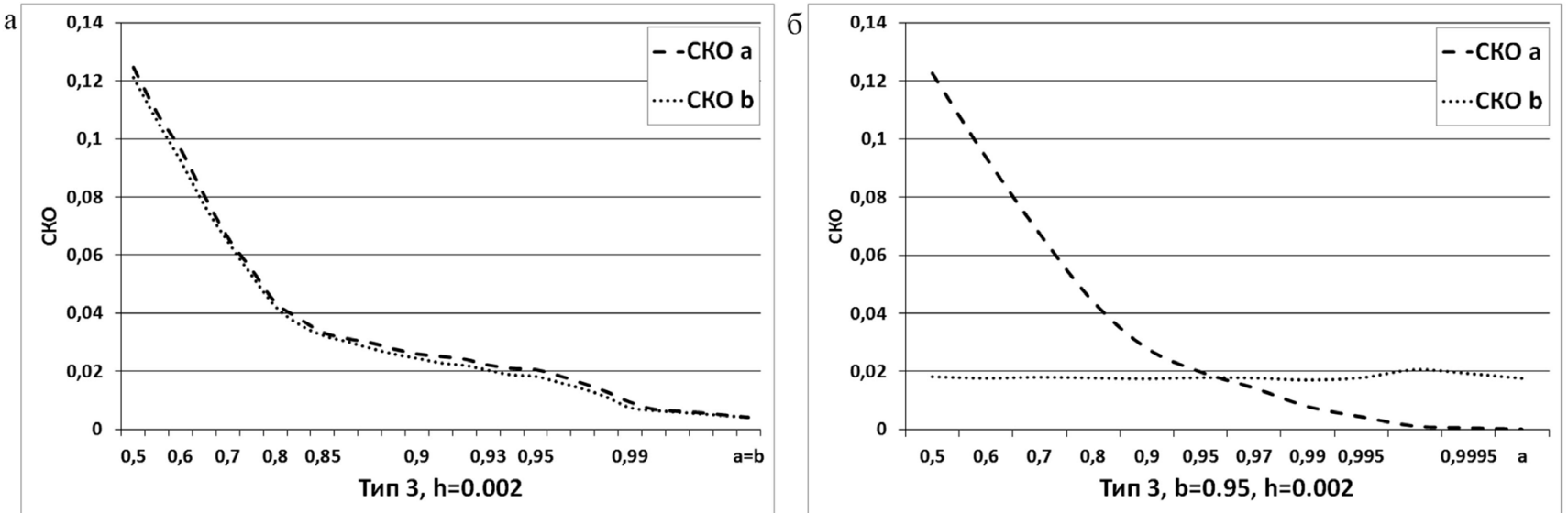

Рис. 17. Тип 3. а) зависимость СКО ошибок от параметров $a=b$; б) зависимость СКО ошибок от параметра $a$ 

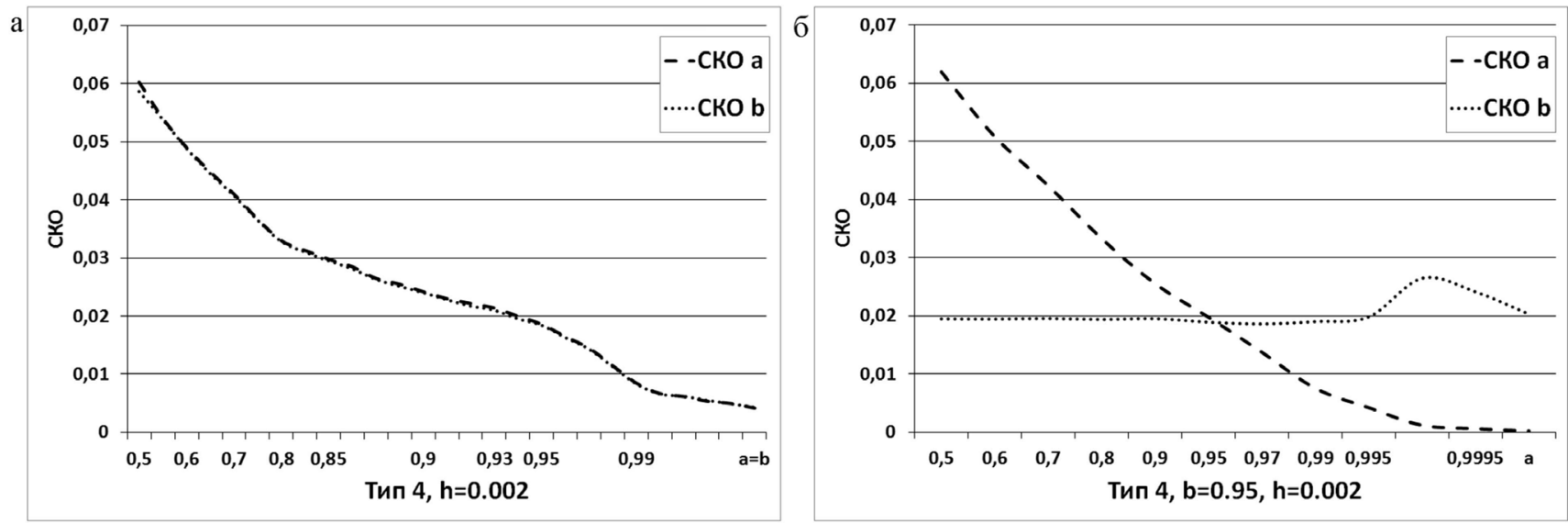

Рис. 18. Тип 4. а) зависимость СКО ошибок от параметров $a=b$; б) зависимость СКО ошибок от параметра $a$
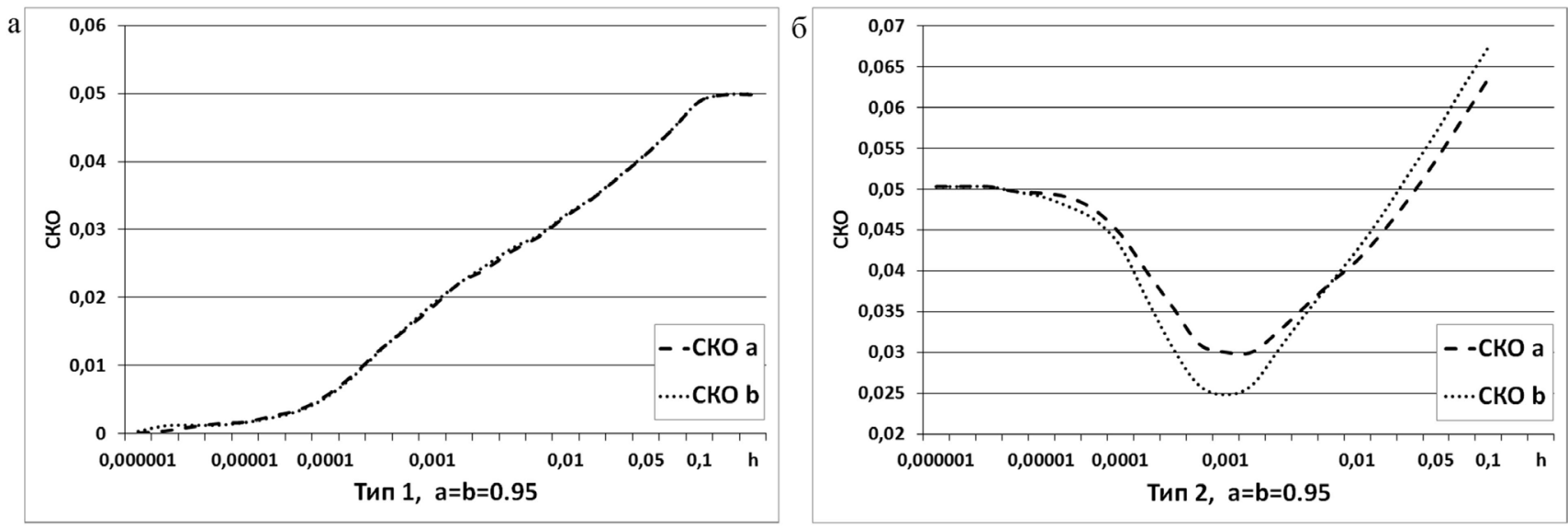

Рис. 19. а) зависимость СКО ошибок от параметра $h$ для Типа 1 ; б) зависимость СКО ошибок от параметра $h$ для Типа 2
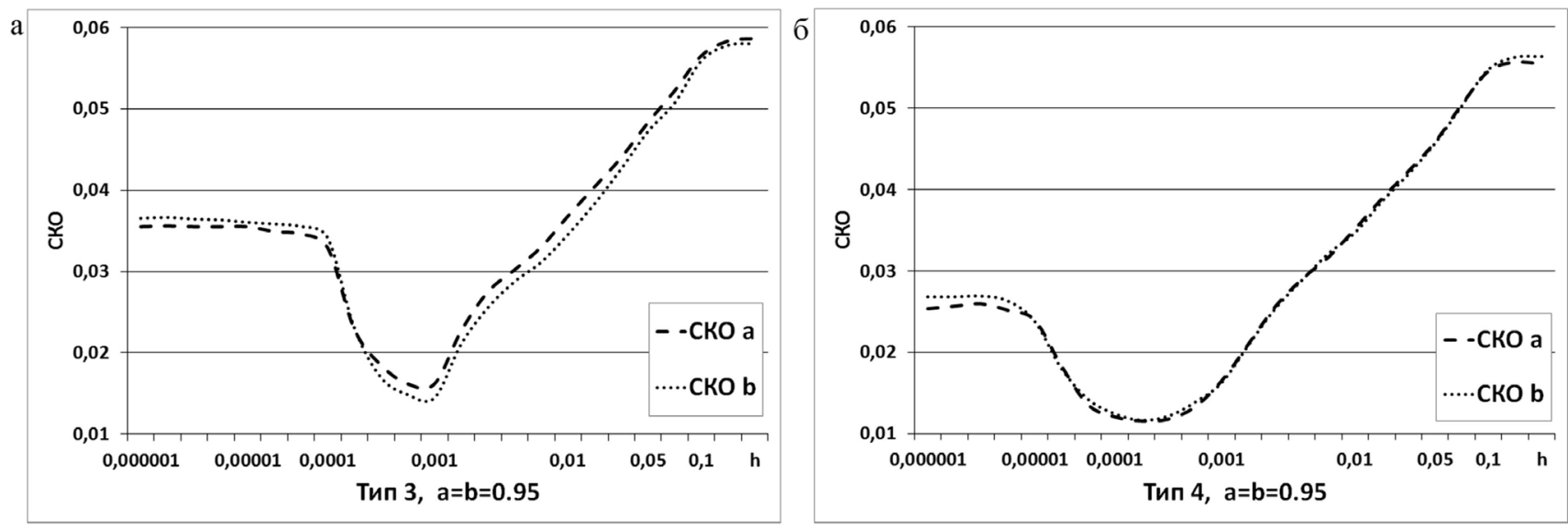

Рис. 20. а) зависимость СКО ошибок от параметра $h$ для Типа 3 ; б) зависимость СКО ошибок от параметра $h$ для Типа 4

Значение параметра $h$ существенно влияет на точность оценки. При его большом значении шаги процедуры велики, поэтому текущие оценки параметров могут значительно отличаться от их точных значений. При малых значениях $h$ текущие оценки параметров могут изменяться медленно, поэтому не успевают отследить изменение оцениваемых параметров. Таким образом, значение $h$ следует принимать в соответствии с классом обрабатываемых изображений. Например, для изображений с постоянными параметрами (тип 1, рис. 19) сле- 
дует брать минимально возможные значения $h$, но для приближения к точным оценкам требуется больше времени. Для изображений с переменными параметрами (типы 2, 3 и 4, рис. 19 и 20) оптимальное значение лежит в интервале $h \in[0,0005 ; 0,002]$.

Зависимость СКО ошибок от характеристик оцениваемых скрытых изображений показана на рисунках 15-18. Естественно, что чем сильнее корреляция скрытых изображений, тем выше точность оценки их параметров.

\section{ЗАКЛЮЧЕНИЕ}

Методы обработки изображений используются для решения многих практических задач. Подавляющее большинство работ по обработке изображений имеют дело с изображениями, заданными на прямоугольных двумерных сетках или сетках более высокой размерности. Иногда изображения определены на цилиндре. Для представления изображений цилиндрических поверхностей предлагаются модели авторегрессии с использованием цилиндрической спиральной сетки пикселей. Спиральное сканирование цилиндрического изображения можно рассматривать как модель квазипериодического процесса. Приведены выражения корреляционной функции изображений в зависимости от параметров модели. Для представления неоднородных изображений со случайными неоднородностями используются дважды стохастические модели, в которых одно или несколько изображений управляют параметрами результирующего изображения. Приведены примеры моделирования изображений четырёх специальных типов управляющих изображений. Управляющие изображения влияют на свойства наблюдаемого результирующего изображения, но сами не наблюдаются. Таким образом, возникает задача идентификации (оценки) скрытых управляющих изображений. Эта задача решается с помощью псевдоградиентных адаптивных алгоритмов. Приведены примеры применения этих алгоритмов и характеристики их точности. В целом можно сделать вывод, что предложенный алгоритм псевдоградиентной идентификации дает достаточно точные оценки параметров.

\section{СПИСОК ЛИТЕРАТУРЫ}

1. Computer Image Processing, Part I: Basic concepts and theory / edited by Victor A. Soifer. VDM Verlag, 2009. 296 p.

2. Morphological image analysis for computer vision applications / Y.V Vizilter, Y.P. Pyt'ev, A.I. Chulichkov, L.M. Mestetskiy // Computer Vision in Control Systems-1. Mathematical Theory. Springer, Cham, 2015. Vol. 73. pp. 9-58.

3. Favorskaya M.N., Jain L.C. Innovative algorithms in computer vision // Computer Vision in Control Systems-4, ISRL, Springer, Cham, 2018. Vol 136. pp. 1-9.
4. Woods J.W. Two-dimensional Kalman filtering // Topics in Applied Physics. Berlin, 1981, Vol. 42. pp. 155-208.

5. Habibi A. Two-Dimensional Bayesian Estimate of Image // Proc IEEE. 1972. Vol 60 (7). pp. 878-883.

6. Gimel'farb G.L. Image Textures and Gibbs Random Fields. Computational imaging and vision. Springer, Kluwer Academic Publishers Dordrecht, 1999. 264 p.

7. Gonzalez R.C., Woods R.E. Digital Image Processing. 4th Ed. Pearson Education, 2018. 1168 p.

8. Bouman C.A. Model Based Imaging Processing. Purdue University, 2013. 449 p.

9. Acharya T., Ray A.K. Image Processing. Principles and Applications. John Wiley \& Sons, 2005. 428 p.

10. Krasheninnikov V.R., Vasil'ev K.K. Multidimensional image models and processing // Computer Vision in Control Systems-3, ISRL, 2018. Vol. 135. pp. 11-64.

11. Krasheninnikov V.R., Kalinov D.V., Pankratov Yu.G. Spiral Autoregressive Model of a Quasiperiodic Signal // Pattern Recognition and Image Analysis. 2001. Vol. 11 (1). pp. 211-213.

12. Krasheninnikov V.R. Correlation Analysis and Synthesis of Random Field Wave Models // Pattern Recognition and Image Analysis. 2015. Vol. 25 (1). pp. 41-46.

13. Dement'iev V.E., Krasheninnikov V.R., Vasil'ev K.K. Representation and Processing of Spatially Heterogeneous Images and Image Sequences // Computer Vision In Control Systems-5. ISRL, Springer, Cham, 2020, Vol. 175. pp. 53-97.

14. Vasil'ev K.K., Dement'ev V.E., Andriyanov N.A. Doubly stochastic models of images // Pattern Recognition and Image Analysis. 2015. Vol 25 (1). pp. 105-110.

15. Поляк Б.Т., Цыпкин Я.З. Псевдоградиентные алгоритмы адаптации и обучения // Автоматика и телемеханика. 1973. № 3. С. 45-68.

\section{REFERENCES}

1. Computer Image Processing, Part I: Basic Concepts and Theory, ed. Victor A. Soifer. VDM Verlag, 2009. 296 p.

2. Vizilter Y.V, Pytev Y.P., Chulichkov A.I., Mestetskiy L.M. Morphological Image Analysis for Computer Vision Applications. Computer Vision in Control Systems-1. Mathematical Theory. Springer, Cham, 2015, vol. 73, pp. 9-58.

3. Favorskaya M.N., Jain L.C. Innovative Algorithms in Computer Vision. Computer Vision in Control Systems-4, ISRL, Springer, Cham, 2018, vol 136, pp. 1-9.

4. Woods J.W. Two-Dimensional Kalman Filtering. Topics in Applied Physics, Berlin, 1981, vol. 42, pp. 155-208.

5. Habibi A. Two-Dimensional Bayesian Estimate of Image. Proc. IEEE, 1972, vol. 60 (7), pp. 878-883.

6. Gimelfarb G.L. Image Textures and Gibbs Random Fields. Computational Imaging and Vision. Springer, Kluwer Academic Publishers Dordrecht, 1999. 264 p.

7. Gonzalez R.C., Woods R.E. Digital Image Processing. 4th Ed. Pearson Education, 2018. 1168 p.

8. Bouman C.A. Model Based Imaging Processing. Purdue University Publ., 2013. 449 p. 
9. Acharya T., Ray A.K. Image Processing. Principles and Applications. John Wiley and Sons Publ., 2005. 428 p.

10. Krasheninnikov V.R., Vasilev K.K. Multidimensional Image Models and Processing. Computer Vision in Control Systems-3, ISRL, 2018, vol. 135, pp. 11-64.

11. Krasheninnikov V.R., Kalinov D.V., Pankratov Iu.G. Spiral Autoregressive Model of a Quasiperiodic Signal. Pattern Recognition and Image Analysis, 2001, vol. 11 (1), pp. 211-213.

12. Krasheninnikov V.R. Correlation Analysis and Synthesis of Random Field Wave Models. Pattern Recognition and Image Analysis, 2015, vol. 25 (1), pp. 41-46.
13. Dementev V.E., Krasheninnikov V.R., Vasilev K.K. Representation and Processing of Spatially Heterogeneous Images and Image Sequences. Computer Vision in Control Systems-5. ISRL, Springer, Cham, 2020, vol. 175, pp. 53-97.

14. Vasilev K.K., Dementev V.E., Andriianov N.A. Doubly Stochastic Models of limages. Pattern Recognition and Image Analysis, 2015, vol. 25 (1), pp. 105-110.

15. Poliak B.T. Tsypkin la.Z. Psevdogradientnye algoritmy adaptatsii i obucheniia [Pseudogradient Algorithms of Adoption and Training]. Avtomatika i telemekhanika [Automatics and Telemechanics], 1973, no. 3, pp. 45-68. 